\title{
EL ESTERO SALADO EN EL DESARROLLO URBANO DE GUAYAQUIL Crónicas de un recurso natural en decadencia
}

\author{
The "Estero Salado" in the urban development of Guayaquil \\ Chronicles of a natural resource in decline \\ Rojas Mosquera, Milton Norberto \\ (IHADIC. Universidad Católica de Santiago de Guayaquil) milromos@gmail.com \\ Rojas Criollo, David Alejandro \\ (IHADIC. Universidad Católica de Santiago de Guayaquil) drojas91@gmail.com
}

\section{RESUMEN}

Debido a su condición de ciudad - puerto, Guayaquil ha sufrido cambios significativos a través de los años. Definida por la importancia del Río Guayas hacia el este, la ciudad se tornó en un punto estratégico para la importación y exportación de productos agrícolas, contando con la infraestructura necesaria para esto (muelles, aduana y plazas de venta e intercambio). Todo este movimiento comercial propició un incremento poblacional y de la trama urbana, principalmente hacia el sur y oeste. Esto generó una conexión con el Estero Salado y sus diferentes ramales, lo que daría a la ciudad una nueva conformación espacial, brindando espacios para actividades de distracción, contemplación y compartir (caza, balnearios y paseos en bote). El estudio de la evolución e impacto del Estero Salado en el desarrollo morfológico de Guayaquil es vital para el entendimiento de las dinámicas socio-espaciales, que han generado una ciudad "moderna" con retos y situaciones complejas.

Palabras clave: asentamientos informales, cuerpos hídricos, promoción inmobiliaria, contaminación ambiental. Bloque temático: teoría e historia de la ciudad.

\section{ABSTRACT}

Due to its status as a port city, Guayaquil has undergone significant changes over the years. Defined by the importance of the Guayas River to the east, the city became a strategic point for the import and export of agricultural products, with the necessary infrastructure for this (docks, customs and places of sale and exchange). All this commercial movement led to an increase in population and urban fabric, mainly to the south and west. This generated a connection with Estero Salado and its different branches, which would give the city a new spatial conformation, providing spaces for activities of distraction, contemplation and sharing (hunting, spas and boating). The study of the evolution and impact of Estero Salado in the morphological development of Guayaquil is vital for the understanding of socio-spatial dynamics, which have generated a "modern" city with complex challenges and situations.

Keywords: informal settlements, water bodies, real estate development, environmental pollution. Topic: theory and history of the city. 


\section{Introducción}

Desde sus inicios, hacia 1547, Guayaquil fue objeto de constantes cambios políticos, sociales y geográficos que han devenido en una discontinuidad de los procesos de conformación y consolidación urbana. El crecimiento acelerado de la ciudad por los auges económicos provocó la ocupación de territorios no aptos para los asentamientos humanos. Esto, sumado a una pobre gestión del cabildo, fue el punto de partida para el progresivo decaimiento del entorno natural.

Las principales afectaciones se dieron en los cerros que rodeaban la ciudad, en las ramificaciones del Río Guayas y de los esteros, que recorrían el perfil oeste de la ciudad. Este proceso de modificación y ocupación del territorio desde inicios del siglo XX es el analizado en el presente estudio.

De esta manera, se pretende caracterizar la presencia del Estero Salado en la evolución de la trama urbana de Guayaquil, junto con los procesos, decisiones y actores que permitieron el decaimiento del cuerpo de agua, y las acciones que se han tomado en tiempos recientes para la recuperación del mismo.

\section{Metodología.}

Para el desarrollo de la investigación se han relacionado enfoques y definiciones sobre los procesos de urbanización y los procesos urbanos (Carrion, 1986), para analizar objetiva y estructuradamente las acciones urbanas producto de las políticas públicas locales. Es además de carácter histórico-cultural y socio-territorial, partiendo de una base analítica descriptiva fundamentada en el levantamiento de información secundaria existente en bibliotecas especializadas en Guayaquil, tesis, publicaciones, relatos históricos y en archivos del Gobierno Local. De igual forma se realizó un acopio de la cartografía histórica permitiendo procesarla digitalmente (mediante software libre de georreferenciación, Qgis) y realizar comparaciones precisas de la evolución de la trama urbana y de sus afectaciones al entorno natural.

Se ha contado además con el apoyo de especialistas (sociólogos, historiadores y arquitectos) que han aportado un análisis histórico-social sobre el crecimiento de la ciudad y cómo las ocupaciones informales han influenciado en éste. La información recabada, permite sistematizar los cambios morfológicos suscitados desde el asentamiento definitivo de la ciudad, con énfasis en las afectaciones que la conformación urbana produjo al Estero Salado.

\section{Antecedentes.}

El asentamiento definitivo de Guayaquil se dio en las faldas del Cerro Santa Ana, con el río Guayas al este, en cuya ribera se extendían tierras bajas e inundables con varias ramificaciones del río. Fue la conexión con éste, y la riqueza forestal en sus inmediaciones, que favoreció la constitución de la ciudad como astillero real para la Corona Española, durante sus primeros 150 años, siendo el "único puerto importante de la Audiencia de Quito" (Laviana Cuetos, 1982).

Debido a que el puerto de Guayaquil sufrió constantes ataques piratas, incendios y pestes, hacia 1688 el Cabildo solicitó, gestionó y obtuvo de las autoridades virreinales, en 1692, la disposición de trasladar la ciudad al sitio de "La Sabaneta", aproximadamente un kilómetro al sur del asentamiento original, donde la Ciudad Nueva adoptó un trazado regular en damero.

La ciudad se desarrolló con los dos asentamientos, separados por una serie de filtraciones del Río Guayas, sobre las que se construyó, en 1710, un puente de madera de 800 varas. Fue un período de transformación urbana y social que produjo un replanteo de los servicios y actividades asentados en las faldas del Cerro Santa Ana. El traslado parcial a "Ciudad Nueva" conllevó un cambio en las dinámicas productivas de la ciudad, debido 
al gran movimiento del comercio de la producción agrícola que reforzó el espacio portuario en la ribera del río Guayas.

Comienza de esta forma el crecimiento de la ciudad, acogiendo a foráneos en busca de oportunidades de trabajo, produciéndose las primeras ocupaciones ilegales de suelo urbano, violando ordenanzas del Cabildo y haciendo uso de tierras de mala calidad (Estrada Ycaza, 1995a).

\section{Presencia del Estero Salado.}

Con un recorrido de más de $50 \mathrm{~km}$, un importante brazo de mar se aproxima a la ciudad desde el sur, presentando diversas ramificaciones a lo largo de su recorrido, siendo las más próximas a Guayaquil el Estero Cobina, el Estero del Muerto, el Estero Santa Ana y el Estero Salado, propiamente. En toda su extensión, los esteros presentan constantemente flujo y reflujo de las mareas oceánicas, razón por la que posee un ecosistema rico en fauna y flora, prevaleciendo el manglar en sus riberas.

Las cuatro ramificaciones mencionadas presentan un recorrido sinuoso que, históricamente, ha establecido los límites naturales de la ciudad hacia el suroeste, los mismos que han ido variando a medida que se rellenaban varias de las prolongaciones de estos esteros hacia la trama urbana en crecimiento no controlado. En la actualidad se aprecian aún los cuatro esteros con una serie de ramales menores que penetran hacia la trama urbana y que se extinguen a unos dos kilómetros del ramal principal (fig. 1).

El Estero Salado se adentra unos 15 kilómetros en la ciudad, llegando hasta la zona central y norte. Estas ramificaciones permitieron disfrutar de un ambiente tranquilo, transformándose en un punto de encuentro por excelencia para los guayaquileños. Incluso se menciona en algunos relatos que las aguas del estero tenían propiedades medicinales (Martillo, 2006),

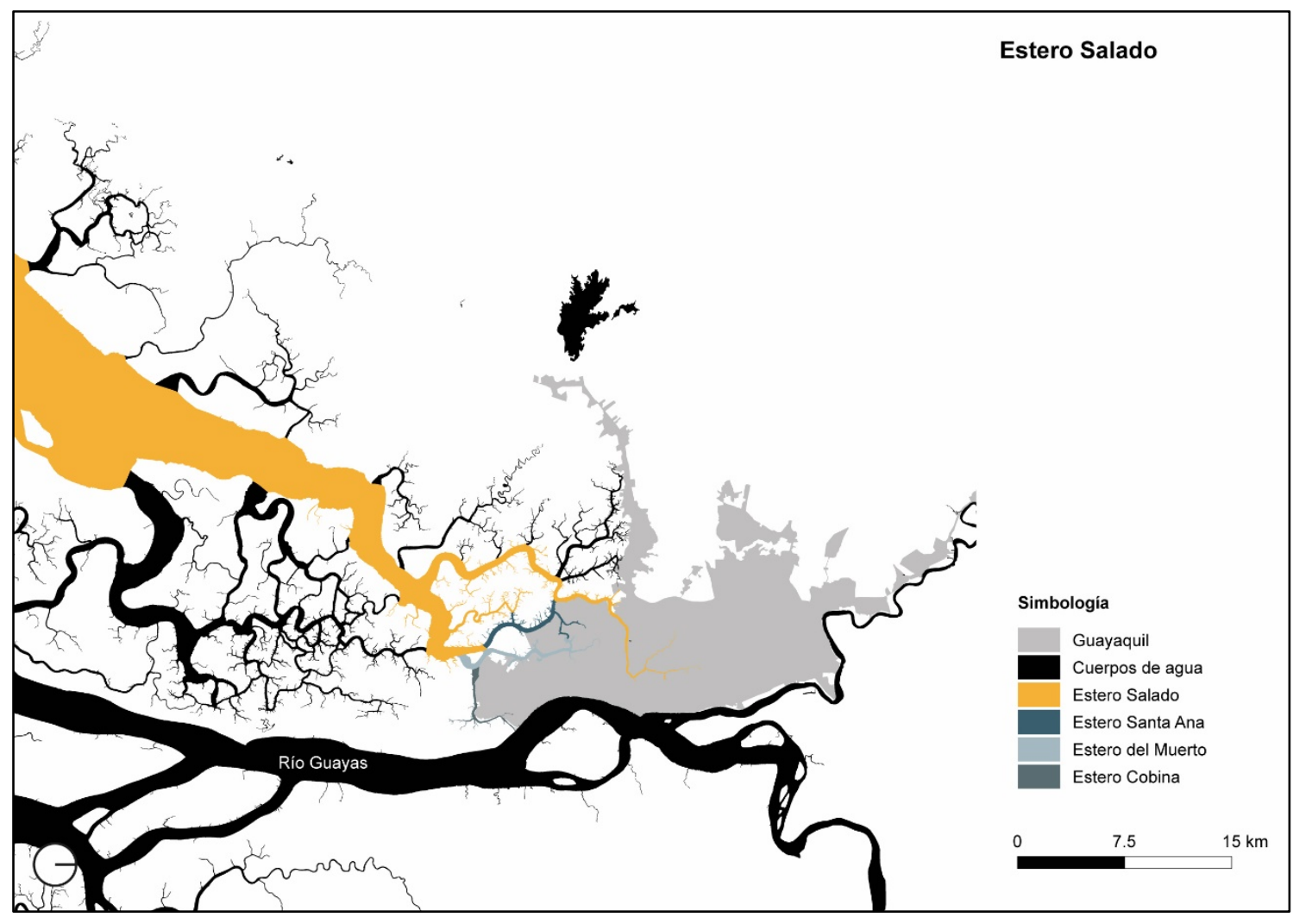

Fig. 1. Esteros que recorren la ciudad. Elaborado por: David Rojas C., mediante software Qgis, en base a cartografía del Instituto Nacional de Estadística y Censos (INEC, 2010). 


\section{1. "Descubrimiento" del Estero Salado.}

La primera fecha en la que consta una descripción sobre el Estero Salado es en 1740 por parte de Jorge Juan, delegado de la Corona Española, que, junto con Antonio de Ulloa, marinos y científicos, fueron parte de la Misión Geodésica Francesa. Entre sus funciones se encontraba la de informar (al Rey) a la Corona Española sobre la situación de los puertos principales de la Costa del Mar del Sur en orden para la mejora del estado de los mismos (Juan \& de Ulloa, 1982). A petición de la Audiencia de Quito, también es encargado de examinar Guayaquil e identificar lo que fuera conveniente para su defensa en caso de ataques vía marítima (Juan \& de Ulloa, 1982). Es así que viaja por el Estero Salado, siendo el primer recorrido técnico-formal del mismo, del que se menciona:

“(...) don Jorge Juan pasó a reconocer todo el Estero Salado, cuyo brazo es tan considerable que, en las cuatro leguas que navegó por él desde la ciudad hasta su boca, encontró siempre 14 brazas de agua, y aún más en algunos parajes" (Juan \& de Ulloa, 1982:157).

Debido a la existencia de terrenos pantanosos entre el asentamiento consolidado de la ciudad y el cuerpo de agua, que dificultaban la accesibilidad, el Estero no será un elemento de importancia para la ciudad hasta un siglo después, a mediados del siglo XIX.

Hacia finales del siglo XVIII la actividad de los astilleros disminuyó, pero a pesar de esto, Guayaquil logró mantener gran prosperidad económica por lo que se conocería como el "Primer Boom Cacaotero", debido a la participación de pequeños y medianos productores (Chiriboga Vega, 2013). La ciudad sufrirá un rápido aumento en la población debido a las migraciones desde la Sierra y el Norte del Perú, como consecuencia del aumento en la producción y exportación cacaotera.

En 1841, Vicente Rocafuerte ${ }^{1}$, como gobernador de Guayaquil, mandó a realizar un corte en el manglar en dirección al oeste y una calzada de cascajo que llevaría al Estero Salado, la que se conoció como el "Camino de la Trocha" (Estrada Ycaza, 1995b).

A inicios del siglo XX, en la zona alrededor del Estero Salado había una gran presencia de manglar, que ocupaba alrededor de 50 metros en las riberas del oeste, hacia el inicio de la cordillera que va a la Costa, y 250 metros al este, hacia la ciudad, seguido por el salitral con un margen de entre 100 y 200 metros (Carbo, 1900) (fig. 2).

\footnotetext{
${ }^{1}$ Fue Presidente de la República del Ecuador entre 1935 a 1939.
} 


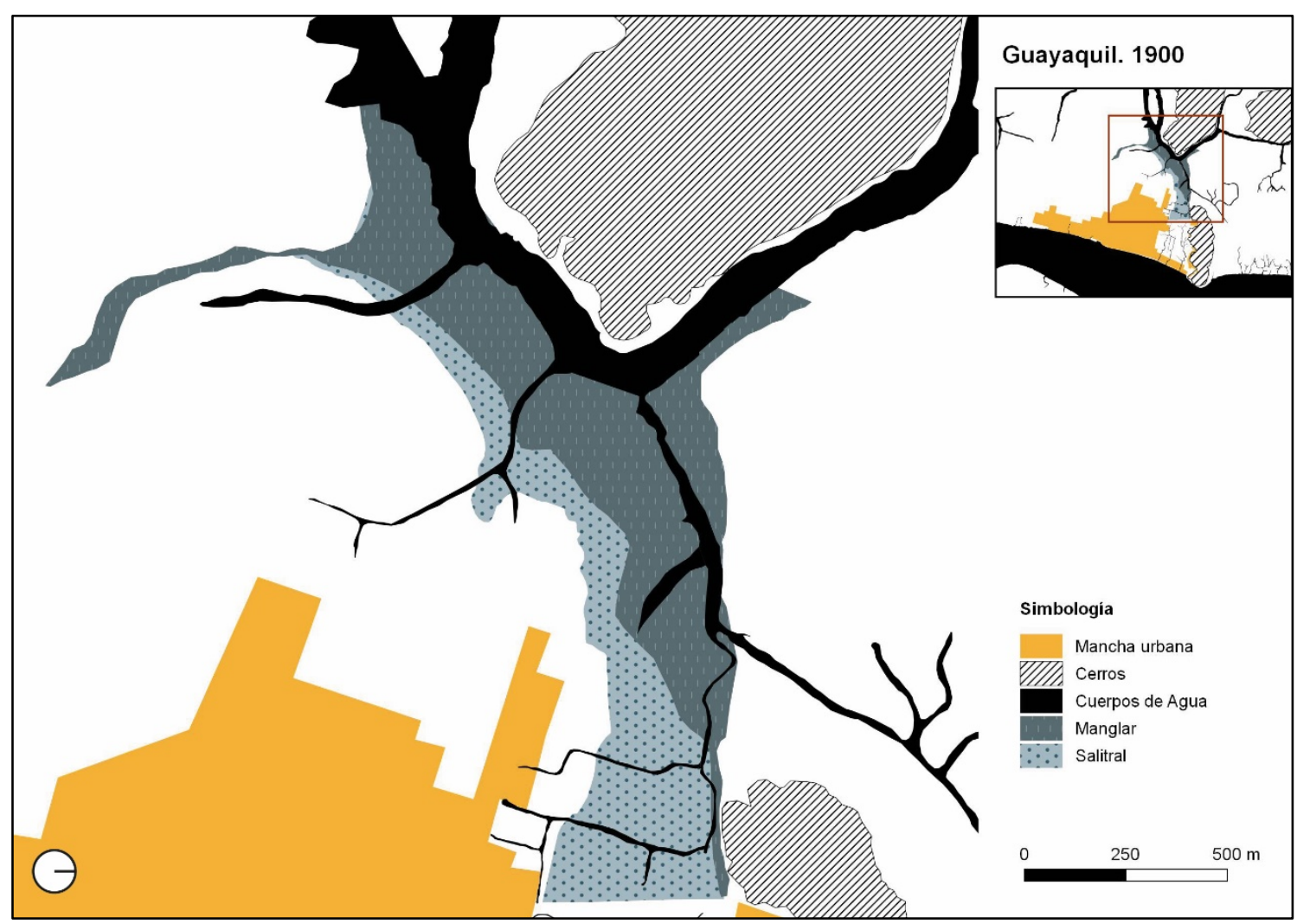

Fig. 2. Manglar y salitral a orillas del Estero Salado (Carbo, 1900). Elaborado por: David Rojas C., mediante software Qgis.

\subsection{Los Baños del Salado.}

Debido a la creciente popularidad del Estero Salado, se construyeron "Los Baños del Salado", un sitio de recreación que paulatinamente se tornó en un gran hito para la ciudad. Hacia el último cuarto del siglo xix, en el Camino de la Trocha circulaban ya carros o vagones halados por mulas, que trasladaban a los habitantes desde y hacia la ciudad. El ambiente en Los Baños era festivo, había música, comida, juegos y deporte (Delgado Cepeda, 2007) (fig. 3).

El complejo contaba con un puente de madera de 104 metros de largo (que alcanzaba a la orilla oeste) desde el que los bañistas realizaban clavadas y piruetas hacia el estero (Estrada Ycaza, 1995b). Guayaquil se vio beneficiado por un nuevo auge económico conocido como el "Segundo Boom Cacaotero" que le permitirá desarrollar proyectos de índole pública.

La población continuó su crecimiento por lo que el Cabildo realizó rellenos de zonas urbanas inundables, en sectores que el río penetraba, y ello a costa de la explotación de canteras aledañas del Cerro El Carmen, límite norte de la ciudad. De igual manera, poco a poco la ciudad se expandió hacia el oeste, aproximándose a la zona de El Salitral y de manglar que bordeaba el Estero Salado. Con todo este crecimiento poblacional Guayaquil, para 1890 ya superó en población a Quito, la capital del país, alcanzando 45.000 habitantes (Hamerly, 2006). 


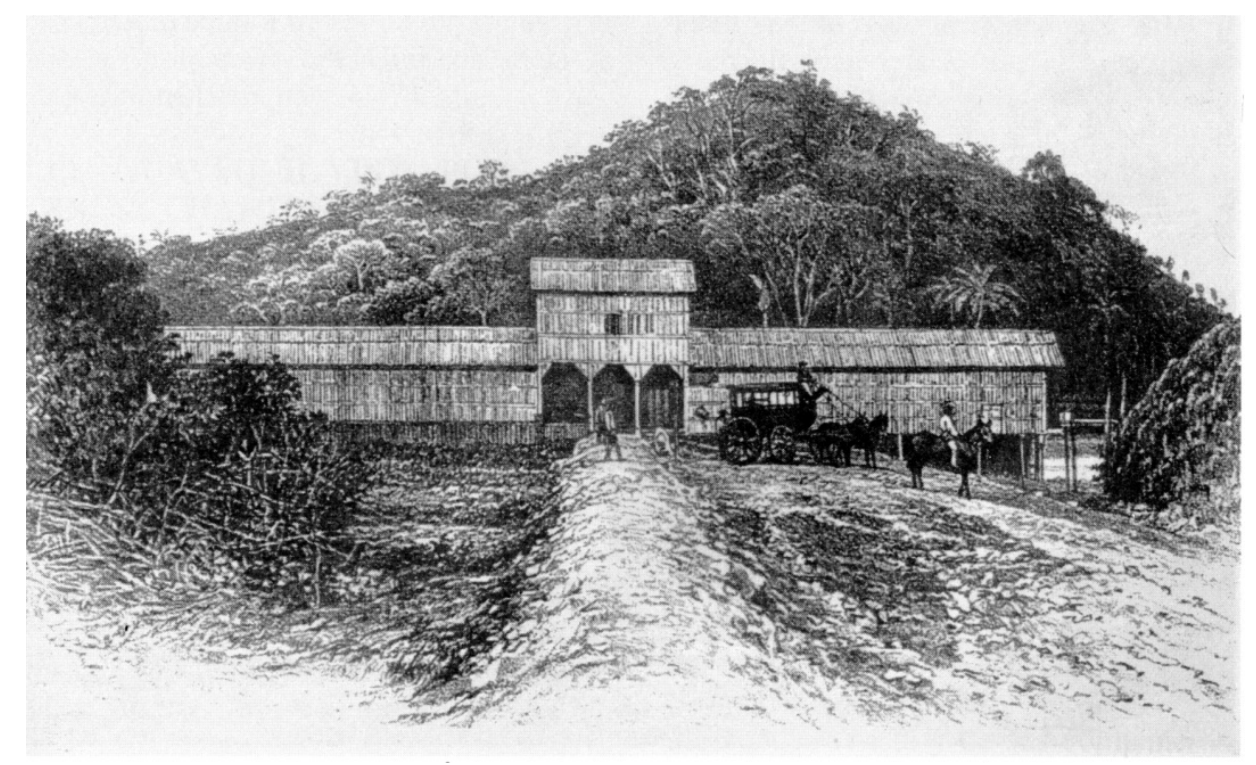

Fig. 3. Ingreso a los Baños del Salado, 1880.(Estrada Ycaza, 1995b).

Los relatos históricos han servido para tener una idea sobre los hechos que fueron modificando el paisaje urbano, así como las vivencias en la ciudad. Esto, junto a los planos históricos indican que Guayaquil poseía una serie de ramales con terrenos anegadizos circundantes, lo que la convertía prácticamente en un pantano en la época invernal y en tiempos de marea alta.

Muchos de los ramales del Estero Salado empezaron a ser cegados a principios del siglo XX, debido a la necesidad de tierra vacante para la creciente población. Esto, acompañado del poco control por parte de las entidades responsables de la ciudad, han sido históricamente las principales causas de la afectación al entorno natural. Entre las principales acciones se identifican la depredación de los cerros para el relleno de las ramificaciones de los esteros y la contaminación de los cuerpos de agua (ya sea por las industrias o los asentamientos humanos).

Siendo un elemento histórico - natural destacado en la constitución de la ciudad, es importante analizar los cambios que ha sufrido en el tiempo, así como las causas y actores que directa o indirectamente tuvieron parte en dicho proceso.

\subsection{Nuevo siglo, nueva ciudad.}

En 1896 Guayaquil enfrentaría el "Gran Incendio", acontecimiento que consumió cerca de dos tercios del centro de la ciudad en las 30 horas que duró (Lee \& Compte, 1993). Como resultado, 92 de las 458 manzanas registradas por el Municipio fueron consumidas, destruyéndose además 1103 casas y edificios de los 4256 existentes (Compte, 2007). Más de la mitad de la población de la ciudad (alrededor de 33000 personas) quedaron sin hogar, teniendo que establecerse temporalmente en los potreros de las quintas aledañas, al norte, y en la zona del Salitral del estero Salado (Carbo, 1900) (ver fig. 6).

A pesar de ser un hecho devastador, tanto social como económicamente, Guayaquil reaccionó prontamente. El Municipio convocó un Concurso de Proyectos Urbanos para la reconstrucción del área afectada, en el que el ingeniero Gastón Thoret, fue elegido para regularizar y prolongar la cuadrícula original, con la menor afectación económica a los ciudadanos, lo que consolidaría el plano urbano de la ciudad (Palacios Jara, 2014), uniendo definitivamente los asentamientos previos de Ciudad Vieja y Ciudad Nueva.

La puntualización de este hecho es necesaria debido al impacto que tuvo sobre la trama de la ciudad, estableciendo la ocupación de nuevas zonas urbanas y contemplando además el relleno de los restantes ramales del Río Guayas y unos pequeños ramales del Estero Salado. Esta forma de habilitar terrenos urbanos 
se convertirá en una acción recurrente para el establecimiento de nuevos asentamientos, significando el sacrificio de los recursos naturales a favor del crecimiento de la ciudad.

Las vertientes aún existentes eran de gran ayuda para la evacuación de aguas lluvias en la temporada invernal, evitando así inundaciones. Es posible apreciar en el plano de Luis Carbo de 1900 la forma en que la ciudad rodeaba las zonas cercanas a los ramales (fig. 4).

En las primeras décadas del siglo XX, la totalidad de las penetraciones del río Guayas, desde el Cerro Santa Ana, hasta el extremo sur de la mancha urbana consolidada, habían sido desecadas, señalándose que la zona de las quintas, al norte, se habilitaría para usos urbanos hacia la década de 1930.

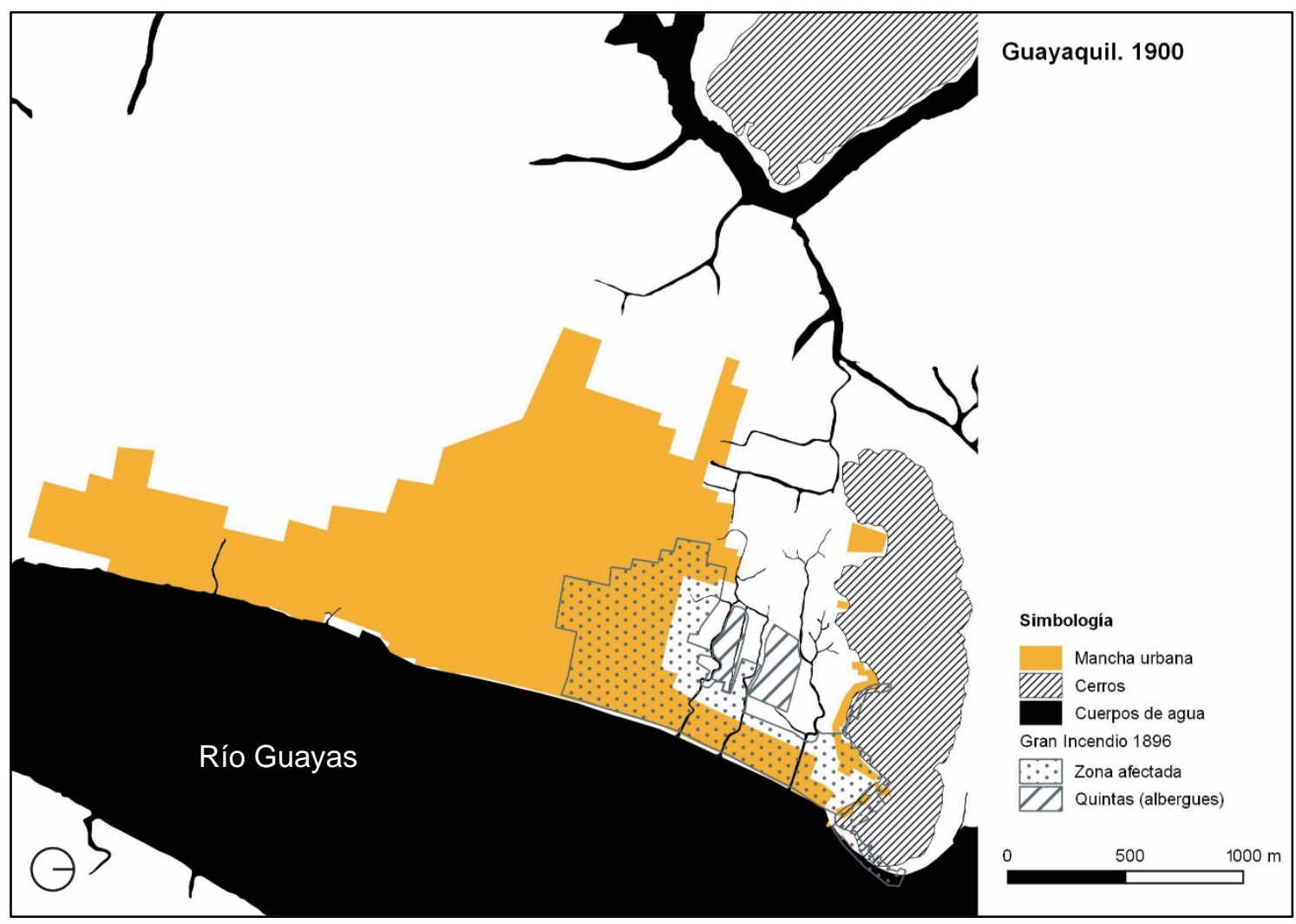

Fig 4. Plano de Guayaquil, 1900 (Carbo, 1900; Thoret, 1896). Elaborado por: David Rojas C., mediante software Qgis.

\subsection{Guayaquil y el centenario de independencia.}

A propósito de los cien años de independencia de la ciudad, a conmemorarse en 1920, y en aras del progreso, en 1912 fue conformada la Junta de Embellecimiento, encargada de velar por la mejora de la imagen urbana de la ciudad; y en 1915, la nueva Junta Patriótica del Centenario planteó una serie de grandes proyectos urbanos, utópicos para la época, que apuntaban a crear una ciudad moderna.

Guayaquil contaba con tranvías eléctricos (1909), una parte de la ciudad estaba pavimentada (1912), y si bien se habían realizado algunas intervenciones para mejorar la salubridad en la ciudad, principalmente para dotación de agua potable, no sería hasta 1914 que el Municipio contrató los servicios de la empresa inglesa J. G. White Company para la realización de obras de saneamiento para toda el área urbana.

En un lapso de pocas décadas, entre 1920 y 1950, los esteros más próximos a la ciudad y a las zonas de expansión inmediatas fueron rellenados, quedando únicamente el cauce principal del Estero Salado y un par de incursiones menores. Cabe mencionar que, a más del relleno, la fauna nativa fue depredada de igual 
manera, desapareciendo gran parte de los manglares ubicados en las riberas de los ramales (fig. 5). La zona principalmente afectada fue la céntrica, como lo narra Julio Estrada Ycaza:

"Ni bien inaugurada la Columna de los Próceres en octubre de 1918, se pudo constatar, a través de una gran laguna que se formó en el siguiente invierno, no solo lo bajo que era el terreno adyacente, sino que la Columna no podía lucirse en la estrecha manzana que se le había asignado originalmente, y se decidió ampliar la Plaza a cuatro manzanas" (Estrada Ycaza, 1995b:108) (fig. 6 y 7).

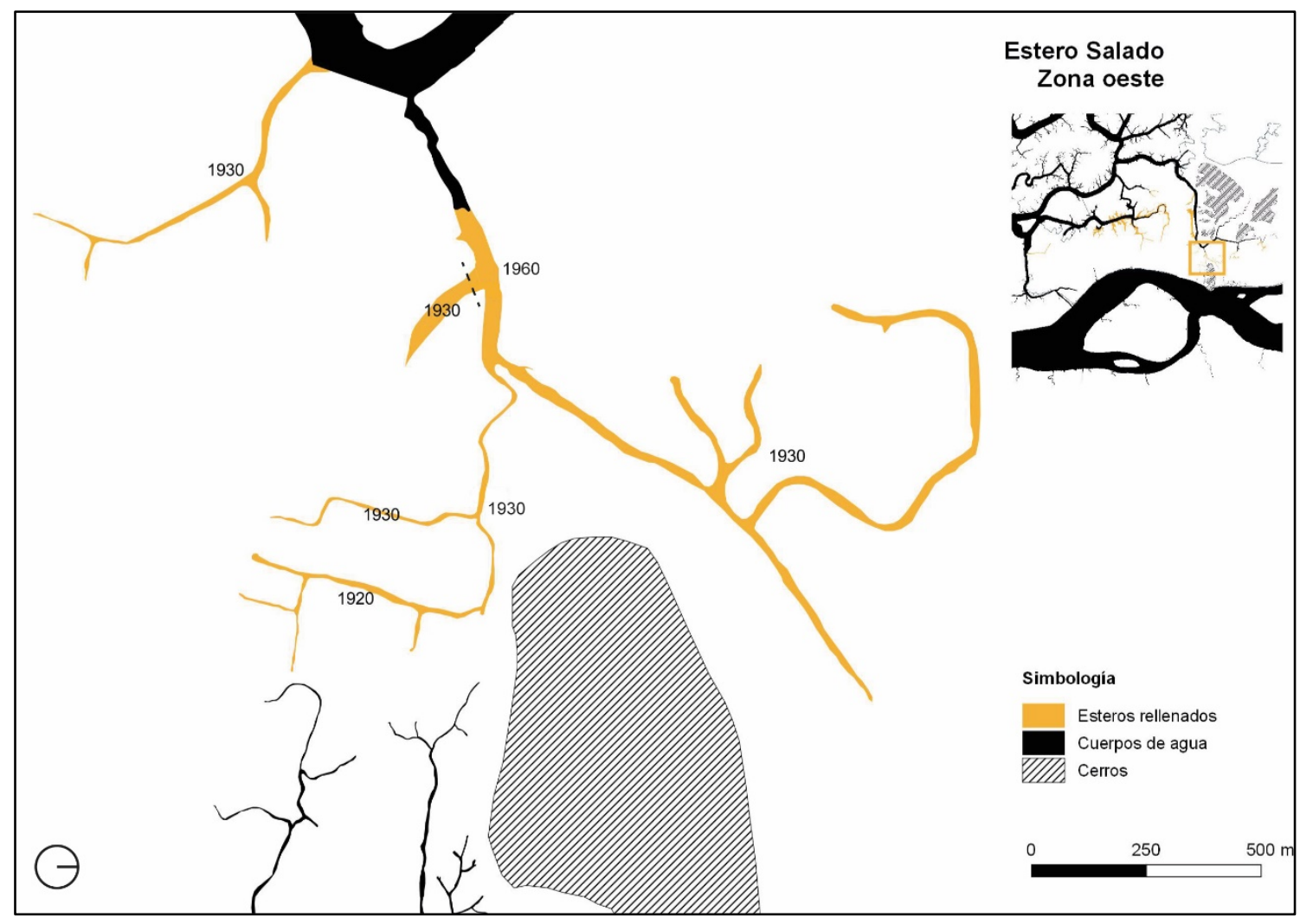

Fig. 5. Identificación de los años en que los ramales del Estero Salado (zona oeste) fueron cegados. Elaborado por: David Rojas C., mediante software Qgis, en base a planos históricos.

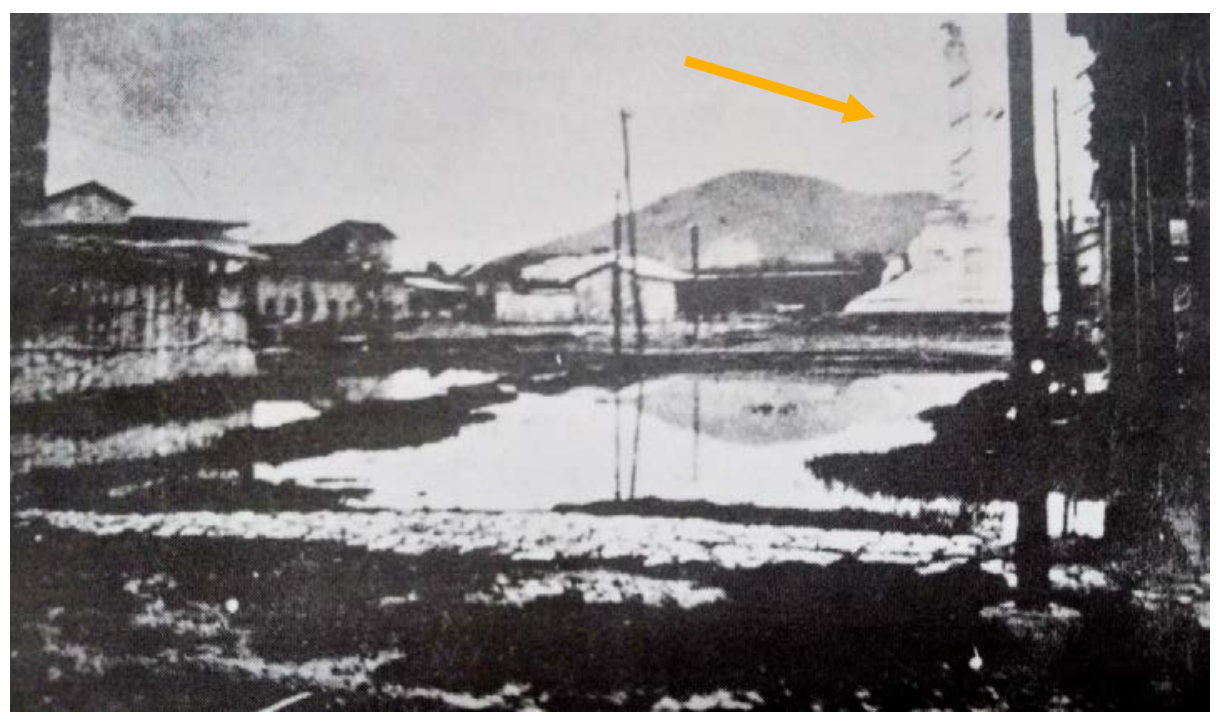

Fig 6. Al fondo a la derecha, la Columna de los Próceres en la pequeña plaza, rodeada de agua debido a una inundación por lluvias, 1919 (Estrada Ycaza, 1995a). 


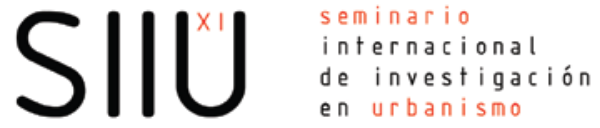

barcelona|santiago dechile junio 2019

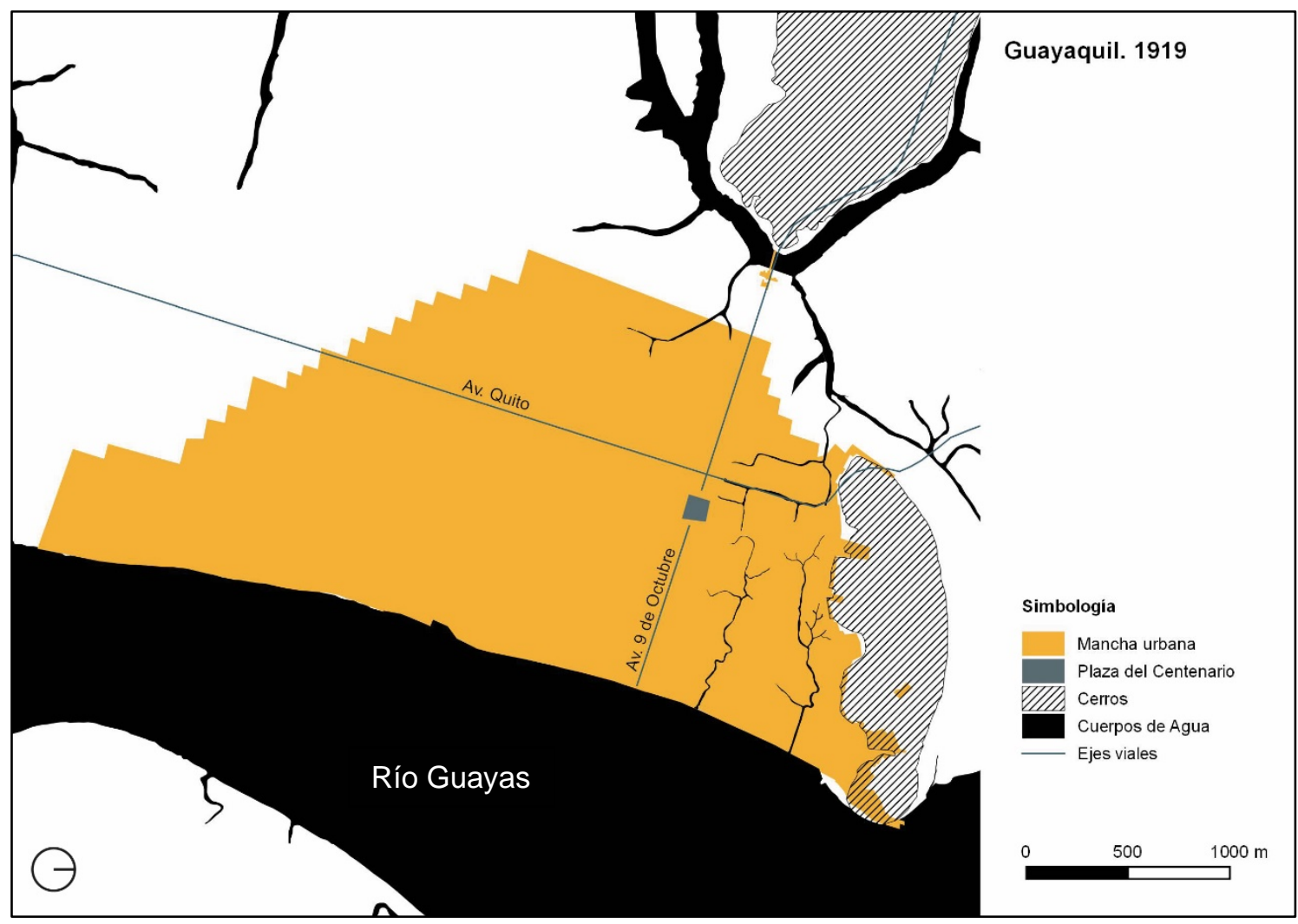

Fig 7. Se puede apreciar la proximidad entre la Plaza del Centenario y los ramales del Salado; además de la separación entre la mancha urbana y los Baños del Salado, 1919 (Holguín, 1919). Elaborado por: David Rojas C., mediante software Qgis.

A pesar de que la mancha urbana no llegaba hasta el Estero Salado, el crecimiento hacia el oeste, como hacia el sur, era continuo. Guayaquil contaba ya con 90.000 habitantes en 1920 (Hamerly, 2006), y la población de escasos recursos que se asentaba en bordes pantanosos, de propiedad municipal, se diversificaba y especializaba, conformada por obreros, trabajadores del tren y de la empresa eléctrica, comerciantes, marineros y cacahueros, lo que la volvía una ciudad de muchas perspectivas.

La bonanza económica, producto del Boom Cacaotero empezó a decaer, tanto por la competencia internacional en el mercado, como por la aparición de plagas que disminuyeron el volumen de producción del cacao. Se vivió una época de incertidumbre que provocó movimientos sociales de consideración, con saldos trágicos en la huelga general de noviembre de 1922, poniendo en evidencia la marcada diferencia social entre la facción pudiente de la ciudad y los trabajadores.

Para 1930 la ciudad enfrentó un proceso de tugurización en la zona céntrica. Las propiedades se subdividieron y fueron arrendadas a la población que llegaba en busca de trabajo. Como consecuencia del hacinamiento y el alto costo de vida, los nuevos citadinos de menores recursos arrendaron al Municipio pequeños lotes en la "Sabana Municipal". En primera instancia, dicha área fue destinada para la construcción temporal de viviendas tras el Gran Incendio de 1896, por lo que el precio de arrendamiento era bajo, lo que fue aprovechado por los nuevos habitantes de la ciudad.

El Ferrocarril a la Costa, en construcción desde 1912, inició sus operaciones parciales desde 1923, llegando a Santa Elena en 1926 y a su destino final, Salinas, en 1935 (Rojas Mosquera, 2019, págs. 95-100), aportando con una conexión más rápida hacia los poblados costeros. La ciudad mantenía un ritmo mercantil constante. Los patios del ferrocarril se encontraban en la orilla este del Estero Salado, alrededor de 300 metros al sur de los Baños del Salado (fig. 8). En los terrenos circundantes se asentaba el personal del ferrocarril, al igual que 
trabajadores que realizaban trabajos en "La Cemento" (empresa dedicada a la extracción de material pétreo y fabricación de cemento en el Cerro San Eduardo, a unos 4 kilómetros de la estación del tren).

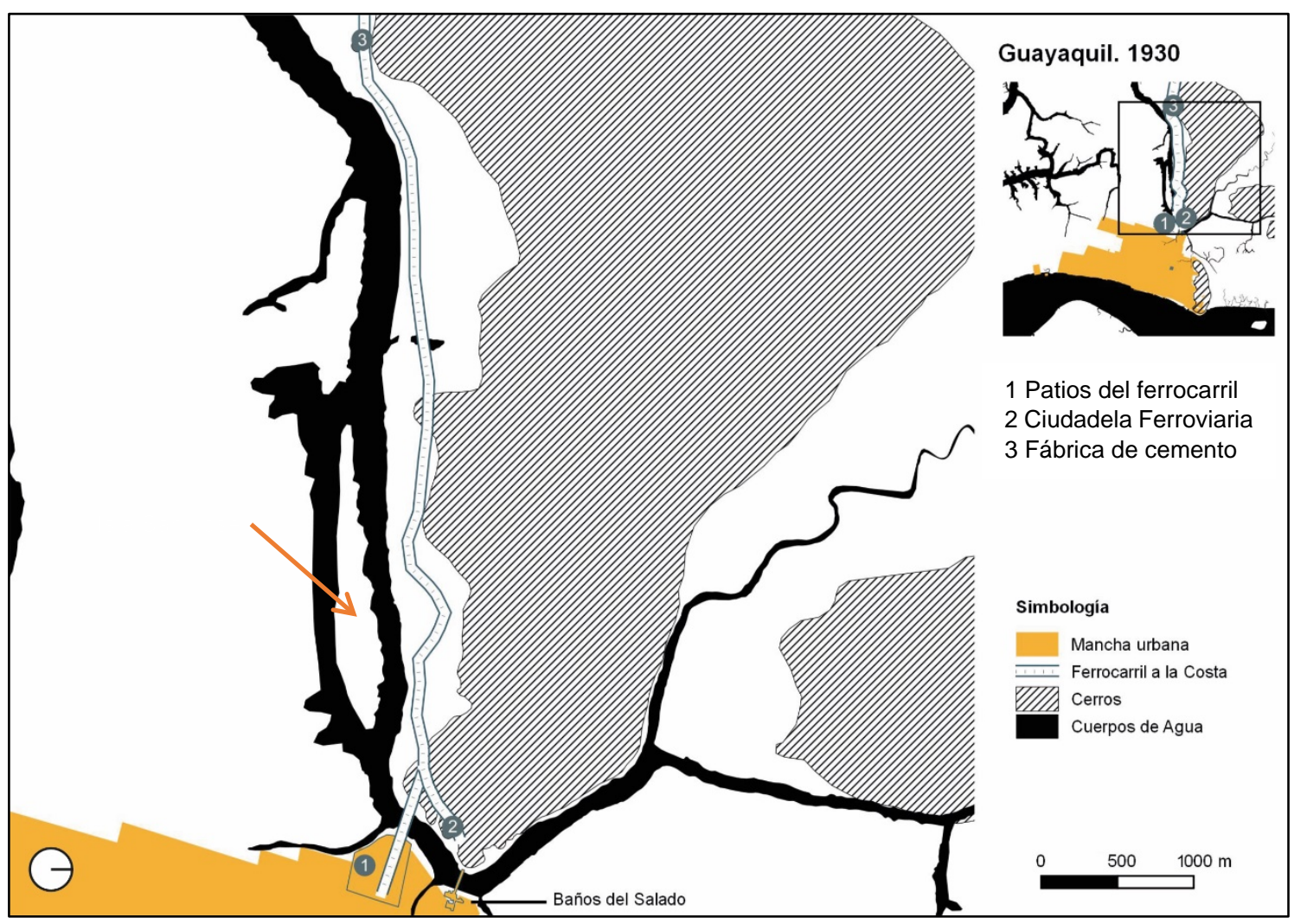

Fig 8. Ruta y paradas del Ferrocarril a la Costa. Elaborado por: David Rojas C., mediante software Qgis, en base a plano de Guayaquil de 1940 (Estrada Ycaza, 1995b).

\subsection{Alto crecimiento de la planta urbana guayaquileña.}

En 1932 se emitió la Ordenanza de Arrendamiento y enajenación de terrenos de propiedad municipal, con la finalidad de alquilar terrenos en los bordes de la "Sabana Municipal" al suroeste de la mancha urbana, y más adelante, entre 1933 y 1941 se hacen reformas a dicha ordenanza, facilitando la ocupación de los manglares municipales y de esa manera, promoviendo una explosiva ocupación del suelo e iniciando una fuerte presencia de asentamientos informales en esta zona, que será conocido como el Suburbio Suroeste. De esta manera, la política urbana, no declarada, de permisividad en la ocupación de pantanos municipales, evitó que los nuevos pobladores se dirijan hacia otras zonas al norte o sur de la ciudad, que eran de propiedad privada y con mejores condiciones de suelo para usos urbanos.

La zona inicial de las grandes ocupaciones de los manglares municipales, se conoció como "Barrio Garay" (en homenaje a un concejal que apoyó tal ocupación) y por esta área el Estero Salado tenía una bifurcación (Estero de la Isla), de aproximadamente un kilómetro y medio, que formaba un pequeño islote. Éste llegó a ser habitado, y posteriormente se volvería parte de "tierra firme" al ser rellenado el canal natural de separación (fig. 9).

Las condiciones de vida en esta zona, debido a ser terrenos pantanosos, era precaria. Las viviendas eran construidas sobre palafitos y no poseían servicios básicos. Se debe mencionar que el material de relleno era extraído de los cerros ubicados tanto al norte como al oeste. Tal fue la magnitud de los rellenos en la ciudad, que de los tres cerros con los que limitaba la ciudad hacia el norte, uno de ellos casi desapareció por completo 
(Cerro de San Lázaro), abriendo calles hacia el norte de los cerros a partir de la década de 1960 (Rojas Mosquera, 2019, pág. 107).

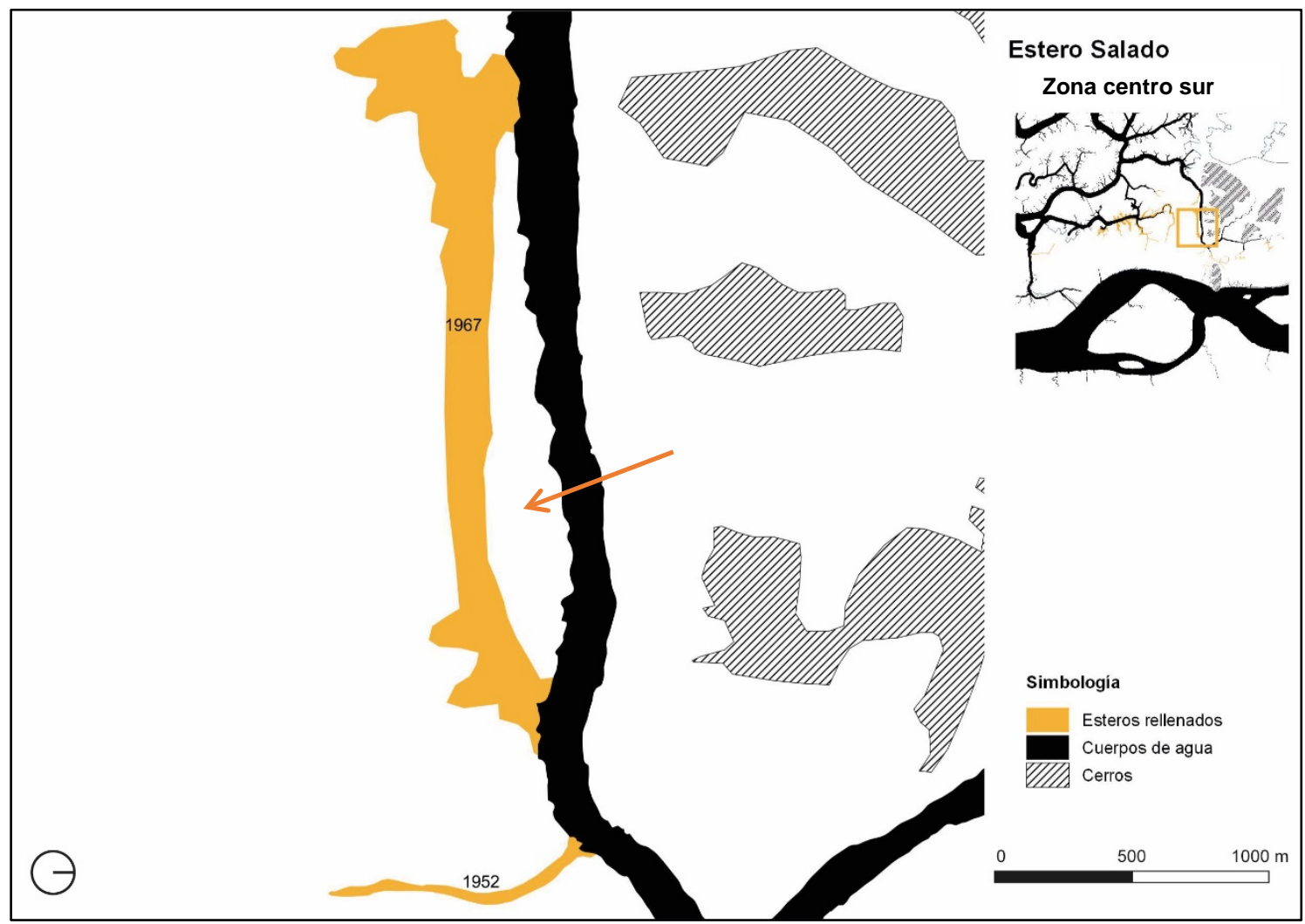

Fig. 9. Identificación de los años en que los ramales del Estero Salado (zona centro - sur) fueron cegados. Elaborado por: David Rojas C., mediante software Qgis, en base a planos históricos.

En 1950 se inauguró la nueva planta de agua potable para Guayaquil, la misma que se encuentra a unos 27 kilómetros al norte de la ciudad en el carretero a Daule. Este hecho produjo la migración masiva de industrias que habían estado asentadas en el sur de la ciudad, las mismas que se alinearon al noroeste, siguiendo dicho eje vial. La contaminación producida desde este sector a partir los años sesenta, ha afectado gravemente a la ciudad, y en particular al Estero Salado, ya que muchos efluentes industriales fueron canalizados, sin tratamiento adecuado, en los últimos tramos del Estero Salado, lo cual produjo una alta contaminación que acabó, en la misma década de 1960, con el área de balneario que había tenido la ciudad por más de cien años (Rojas Mosquera, Villavicencio, Chang, \& Bécquer, 1989, pág. 45 y ss) y con varios ecosistemas en las zonas cercanas a esas fábricas.

A mediados de la década de 1950, en un sector a menos de 2 kilómetros al noroeste de lo que era el balneario de El Salado, el "American Park", la inversión privada ejecutó la más grande inversión, hasta esa época, en un programa residencial para clases media y alta, que se denominó URDESA (Urbanizadora del Salado S.A.), el mismo que estaba bordeado por ramales del Salado. Los terrenos entre dichos ramales eran bajos y pantanosos, por lo que fueron rellenados en una superficie de más de 200 hectáreas, a costa de cerros que limitaban esta área, al norte. De esta manera, la afectación ambiental de esta habilitación urbanística, en menos de 5 años de trabajos, acabó con la fauna y flora de todo este amplio sector aledaño a la ciudad.

En 1962 el Puerto Marítimo que se había desarrollado linealmente en el Malecón de la ciudad, es trasladado hacia el extremo sur de la ciudad (en la ribera del Estero del Muerto), generando un nuevo eje de crecimiento alrededor de los 6 kilómetros de la carretera de acceso desde la ciudad, donde se ubican algunas fábricas, así 
como diversos programas de vivienda promovidos por el Estado Central que se ejecutaron en la década de 1970 (fig. 10).

A mediados de los 70, se promulgó un Esquema Preventivo para el Plan de Desarrollo Urbano en el que se proponía la expansión de la ciudad hacia el sur. Esto se vio truncado por la ocupación masiva de terrenos (entre 1975 y 1982) en sectores del sur, conocido como El Guasmo. La magnitud de estos asentamientos informales fue tan fuerte, que forzaron un nuevo eje de crecimiento de la ciudad, ya que los inversores (tanto privados como públicos) buscaron nuevas zonas para promoción inmobiliaria, trasladándose hacia el norte de la ciudad. Los programas de vivienda privados se ubicaron en zonas alejadas de la mancha urbana, al noroeste del aeropuerto, y poco después, múltiples programas de vivienda del estado se asentaron junto a ellos, a partir de la década de 1980, señalándose que hacia esta zona no existía la presencia de ramales del Estero Salado.

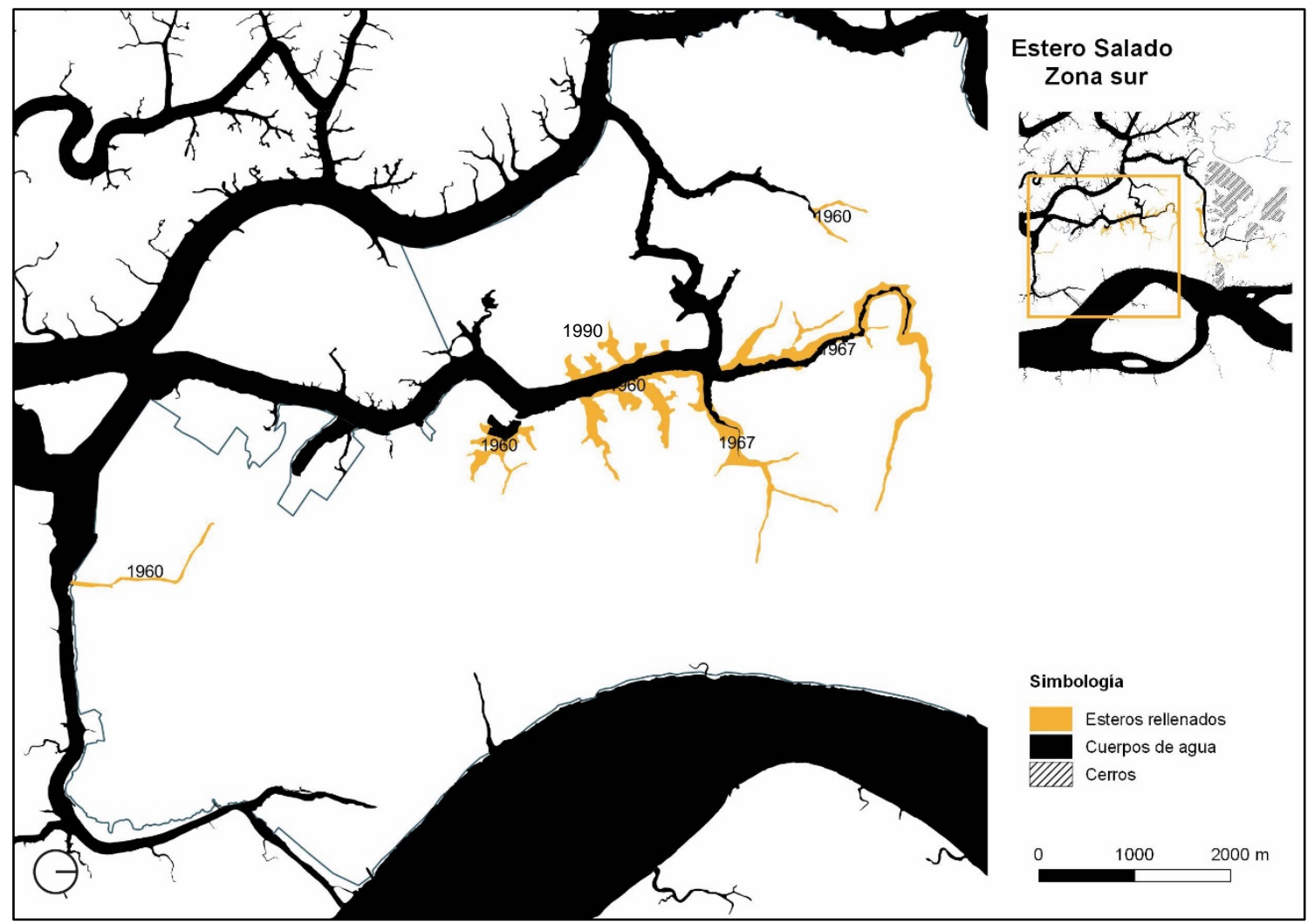

Fig. 10. Identificación de los años en que los ramales de los esteros Santa Ana y Del Muerto, al sur de la ciudad, fueron cegados. Elaborado por: David Rojas C., mediante software Qgis, en base a planos históricos.

La ocupación del norte de la ciudad, cercana al eje de la vía a Daule, afectó de igual manera a los ramales del Estero Salado. La promoción inmobiliaria privada, intervino en los cerros de los sectores aledaños y los ramales circundantes (fig. 11).

Al no existir por parte de la Municipalidad una planificación estructurada y legalmente establecida que permitiera realizar un estricto control sobre los procesos de urbanización y habitabilidad en la ciudad, la contaminación de los ramales y del Estero Salado (particularmente) fue constante y progresiva. La disposición de los desechos inorgánicos y orgánicos, era realizada directamente hacia este recurso hídrico, tanto por las industrias de la zona de Mapasingue en la vía a Daule, como de los sectores populares irregulares aledaños, pero también desde las nuevas urbanizaciones formales, como URDESA y Miraflores (colindantes al noroeste con la zona de Mapasingue), todo lo cual contribuyó al galopante proceso de degradación ambiental. 


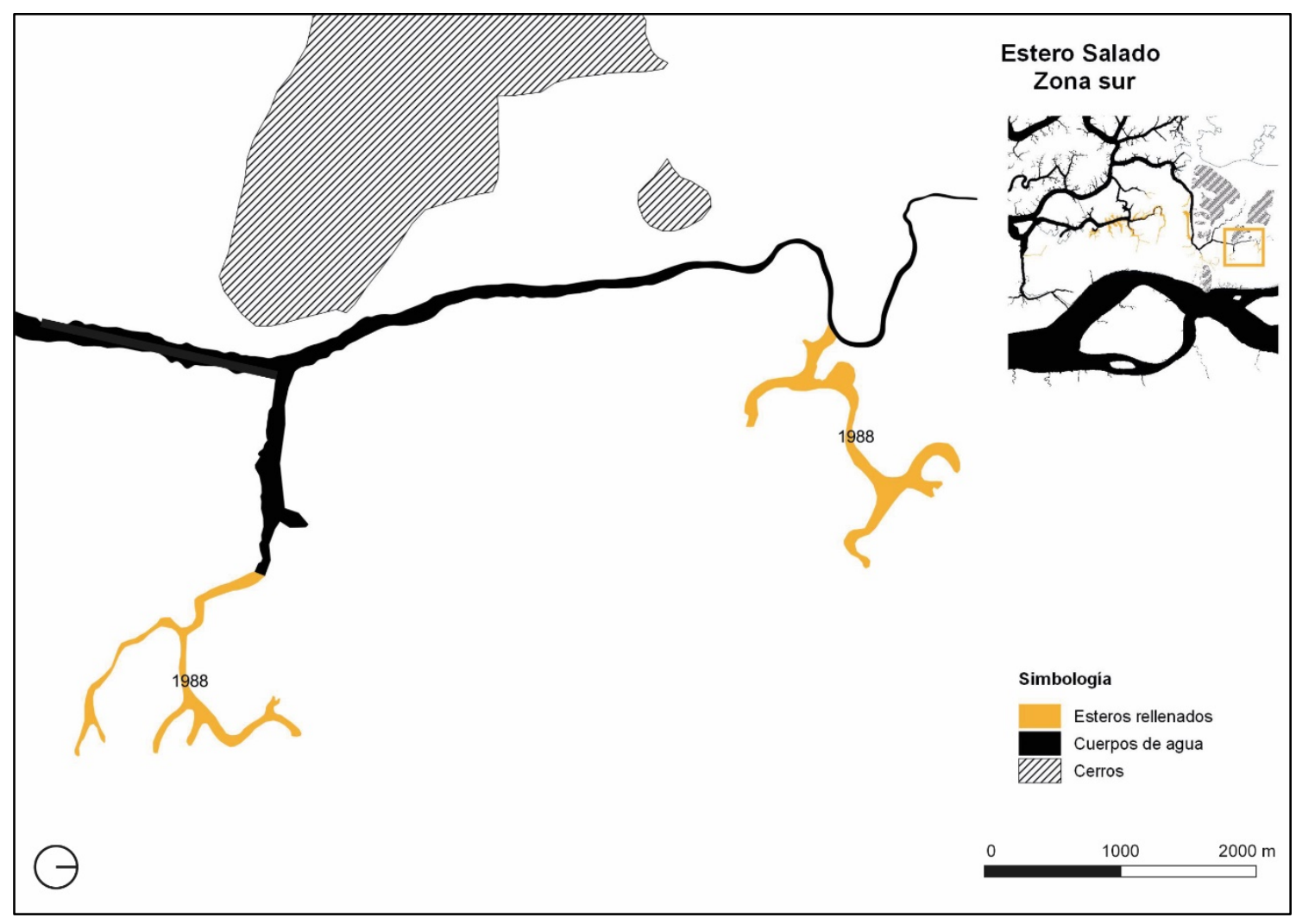

Fig. 11. Identificación de los años en que los ramales del Estero Salado, en el sector norte, fueron cegados. Elaborado por: David Rojas C., mediante software Qgis, en base a planos históricos.

\subsection{De vuelta al Estero.}

A pesar del mal logrado en el siglo XX, en años recientes se han desarrollado esfuerzos y acciones para la recuperación del Estero Salado. Iniciativas privadas, públicas y de la sociedad civil han surgido, promoviendo una nueva visión y cultura de los esteros.

Las regulaciones por parte del Municipio se han vuelto más estrictas. Desde julio de 2000, existe legalmente un Plan Regulador Urbano de Guayaquil, el mismo que se ha ido actualizando y complementando con ordenanzas específicas. Aun así, siguen existiendo casos de industrias que desalojan los desechos directamente al Estero Salado, al igual que las zonas residenciales populares ubicadas en las riberas de los ramales. Cabe indicar que el Alcalde Jaime Nebot, en funciones continuas entre los años 2000 y $2019^{2}$, en noviembre de 2018 reconoció que una deuda de su gestión era la falta de recuperación ambiental en el Estero Salado (El Universo, 2018).

Por su parte, en años anteriores, el Estado Central generó un macro plan para Guayaquil (y su área de influencia), conocido como Guayas Ecológico, dentro del cual se encuentra el proyecto "Recuperación Ecológica del Estero Salado" que promueve principalmente un impulso a la Reserva de Producción de Fauna Manglares del Salado.

"Proporcionar a los habitantes de la ciudad de Guayaquil de áreas verdes para la recreación, esparcimiento, contacto con la naturaleza y fomentar la generación de servicios ambientales permanentes en la urbe" («Guayaquil Ecológico», 2012)

\footnotetext{
${ }^{2}$ Nebot culminó su mandato el 14 de mayo de 2019.
} 
Bajo este objetivo, el proyecto establece la recuperación de 41 kilómetros de riberas, divididos en 11 tramos, mediante intervenciones como parques lineales, zonas deportivas y áreas de descanso (fig. 12). Además del desarrollo de procesos técnico - científicos y de promover la educación ambiental y social.

Para la implementación del proyecto fue necesario el traslado y reubicación de 3478 familias que vivían en las riberas del Estero Salado, hasta nuevos programas de vivienda estatal, distantes de este sector, con el consiguiente malestar social de las familias afectadas. Paralelamente, se implementó la limpieza de cerca de 10 kilómetros de riberas (Ministerio de Desarrollo Urbano y de Vivienda, 2015).

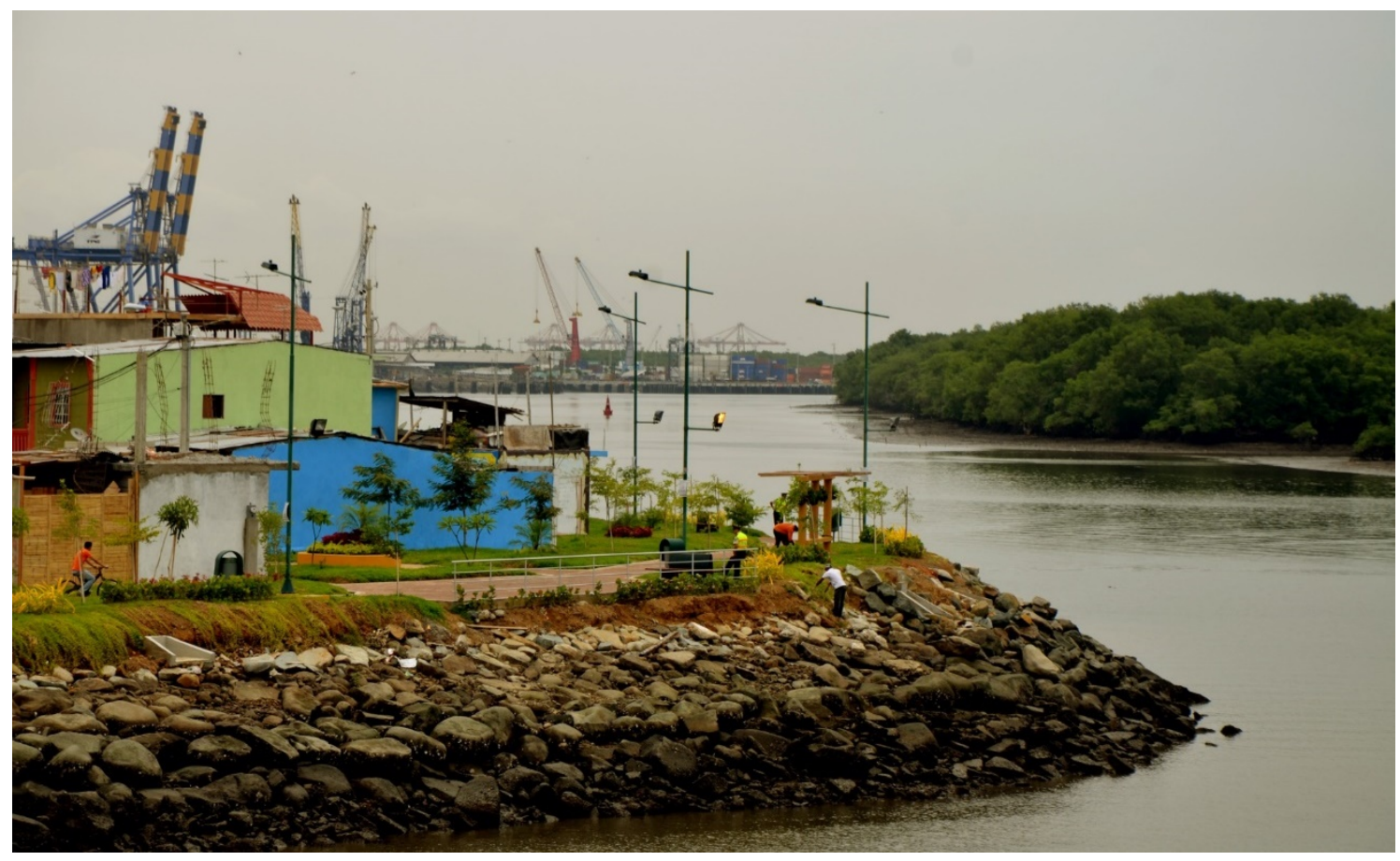

Fig. 12. Intervención en riberas del Estero Salado, 2013.

\section{Conclusiones}

Ante la evidencia recolectada y el análisis de la misma, se puede contemplar que la gestión del cabildo (municipio), salvo puntuales ocasiones, no ha logrado implementar los instrumentos técnicos y legales necesarios que orienten el crecimiento urbano de Guayaquil, evidenciando en el proceso la pésima calidad de habitabilidad a la que han tenido que resignarse los asentamientos populares irregulares en las áreas periurbanas de la ciudad.

La inversión inmobiliaria pública, ha tenido la tendencia de desarrollar los programas de vivienda social en las periferias urbanas, como pudo ser constatado a partir de la década de 1960. En una aproximación similar, la promoción inmobiliaria privada en las décadas de los 50, 70 y 80 ha sido cómplice en un proceso de depredación extenso, habilitando grandes zonas urbanas periféricas, sin tener en cuenta el negativo impacto causado a los elementos urbanos naturales que poseía la ciudad.

En todo este proceso histórico, social y cultural, no ha existido una conexión real entre todas las partes interesadas en la conservación del Estero Salado, por lo que se hace necesario llegar a dicha relación para poder contar nuevamente con un cuerpo de agua vivo, que posibilite actividades urbanas de movilidad y distracción, promoviendo un desarrollo urbano integral, que sólo podrá ser logrado con un trabajo conjunto y continuo. Por estas consideraciones, corresponde aunar esfuerzos de los estamentos públicos nacionales y locales, academia, organizaciones sociales y de las empresas privadas para la búsqueda y aplicación de acciones que garanticen la sostenibilidad de los recursos naturales en el contexto guayaquileño. 


\section{BIBLIOGRAFÍA.}

\section{Obra completa}

BOCK, S. (1992). Guayaquil. Arquitectura, espacio y sociedad. 1900 - 1940. Guayaquil: IFEA CERG

CARBO, L. A. (1900). Plano de la ciudad de Guayaquil. Histórico, Guayaquil, Ecuador.

COMPTE, F. (2007). Arquitectos de Guayaquil. Guayaquil, Ecuador: UCSG FAD.

HOLGUín, F. (1919). Plano de la ciudad de Guayaquil. Histórico, Guayaquil, Ecuador.

LEE, P., \& COMPTE, F. (1993). Guayaquil: lectura histórica de la ciudad. Forma urbana y propuesta de ciudad desde su fundación. Guayaquil, Ecuador.

ROJAS MOSQUeRA, M., y VILLAVICENCIO, G. (1988). El Proceso Urbano de Guayaquil. 1870 - 1980. Guayaquil: ILDIS CERG.

ROJAS MOSQUERA, M., VILLAVICENCIO, G., CHANG, L., \& BÉCQUER, A. (1989). El mercado de suelo urbano y barrios populares en Guayquil. Guayaquil: CERG - IDRC.

ROJAS MOSQUERA, M. (2019). El proceso urbano de Guayaquil. Del espacio portuario a la metrópoli (En prensa) (Instituto de Hábitat, Diseño y Construcción, Ed.) Guayaquil, Ecuador: Universidad Católica de Santiago de Guayaquil.THORET, G. (1896). Plano de la ciudad de Guayaquil. Histórico, Guayaquil, Ecuador.

Capítulo de libro

CHIRIBOGA VEGA, M. (2013). Jornaleros, grandes propietarios y exportación cacaotera, 1790-1925 (2. ${ }^{a}$ ed.). Quito, Ecuador: Corporación Editora Nacional.

JUAN, J., y DE ULLOA, A. (1982). Noticias secretas de América. Madrid: Turner.

PALACIOS JARA, A. (2014). Gastón Thoret, gestor de la reconstrucción de Guayaquil después del Incendio Grande, 1896. En Ing. Gastón Thoret Jager. Ingeniero, constructor y visionario (Primera). Guayaquil, Ecuador.

Varias obras del mismo autor

ESTRADA YCAZA, J. (1995a). Guía histórica de Guayaquil (Vol. 2). Guayaquil: Banco del Progreso. (1995b). Guía histórica de Guayaquil (Vol. 3). Guayaquil: Banco del Progreso.

Revistas

HAMERLY, M. T. (2006). Recuentos de dos ciudades: Guayaquil en 1899 y Quito en 1906. Procesos. Revista ecuatoriana de historia, 1(24), 135-163.

LAVIANA CUETOS, M. L. (1982). Una descripción inédita de Guayaquil. Temas Americanistas, 12.

Fuentes electrónicas

Delgado Cepeda, H. (2007, octubre 4). Los baños del Salado. El Universo. Recuperado de https://www.eluniverso.com/2007/10/04/0001/18/A39C9F3749764A4FBA1995B02DFFBFB7.html

Guayaquil Ecológico. (2012, octubre 31). Recuperado 10 de marzo de 2019, de http://www.ambiente.gob.ec/guayaquil-ecologico/

INEC. (2010). Cartografía digital 2010 [Recursos digitales nacionales de Ecuador]. Recuperado de http://www.ecuadorencifras.gob.ec/cartografia-digital-2010/ 
Laviana Cuetos, M. L. (1987). Guayaquil en el Siglo XVIII: Recursos Naturales y Desarrollo Económico. Sevilla: Escuela de Estudios Hispano-Americanos de Sevilla, C.S.I.C. Recuperado de https://books.google.com.ec/books?id=cSp6AAAAMAAJ

Martillo, J. (2006, mayo 30). De baños curativos al Malecón del Salado. El Universo. Recuperado de https://www.eluniverso.com/2006/05/30/0001/18/43D29C596CA549C0A4BFD1200BA692C1.html

Ministerio de Desarrollo Urbano y de Vivienda. (2015). Generación de áreas verdes y espacios recreativos públicos para la provincia del Guayas - Guayas Ecológico.

5. 